\title{
Orofacial Granulomatosis: Report of a case
}

\author{
Yoshinori Jinbu ${ }^{1}$, Keiichi Tsukinoki ${ }^{2}$, Mikio Kusama ${ }^{1}$ and Yoshihisa Watanabe ${ }^{2}$ \\ ${ }^{1}$ Department of Dentistry and Oral Surgery, Jichi Medical School, Tochigi , Japan \\ ${ }^{2}$ Department of Oral Pathology, Kanagawa Dental College, Kanagawa, Japan
}

Jinbu Y, Tsukinoki K, Kusama M and Watanabe Y. Orofacial granulomatosis: Report of a case. Oral Med Pathol 2002; 7: 33-37, ISSN 1342-0984.

A case of orofacial granulomatosis with buccal mucosal lesion is reported. The patient was a 44-year-old Japanese male who presented with a nodular lesion in the buccal mucosa. Pathology of the resected specimen showed a non-caseating granuloma suggesting Crohn's disease or sarcoidosis. Systemic and biochemical examination showed no evidence of Crohn's disease or sarcoidosis. Finally, based on clinical features and pathological findings, we diagnosed the patient as having orofacial granulomatosis.

Key words: Orofacial granulomatosis, Crohn's disease, sarcoidosis, non-caseating granuloma Correspondence: Yoshinori Jinbu, Department of Dentistry and Oral Surgery, Jichi Medical School, Yakushiji 3311-1, Minamikawachi-machi, Kawachi-gun, Tochigi, 329-0498, Japan Phone: +81-285-58-7390, Fax:+81-285-44-8669

\section{Introduction}

Orofacial granulomatosis is a clinical entity presenting as a chronic swelling of the oral or facial tissues in association with histological evidence of non-caseating granulomatous inflammation (1). The term orofacial granulomatosis is familiar in North America, Europe, Australia and Africa, and there have been many case reports and reviews in those countries (2-8). However, the term is not common in Japan. In most cases of orofacial granulomatosis, the etiology remains unclear, although some cases are associated with Crohn's disease or sarcoidosis. A few patients seem to have specific intolerances to foods or additives (9-11). Therefore, careful diagnostic assessment is needed for these patients.

This case report describes a patient who presented with a nodular lesion in the buccal mucosa and who was finally diagnosed as having orofacial granulomatosis.

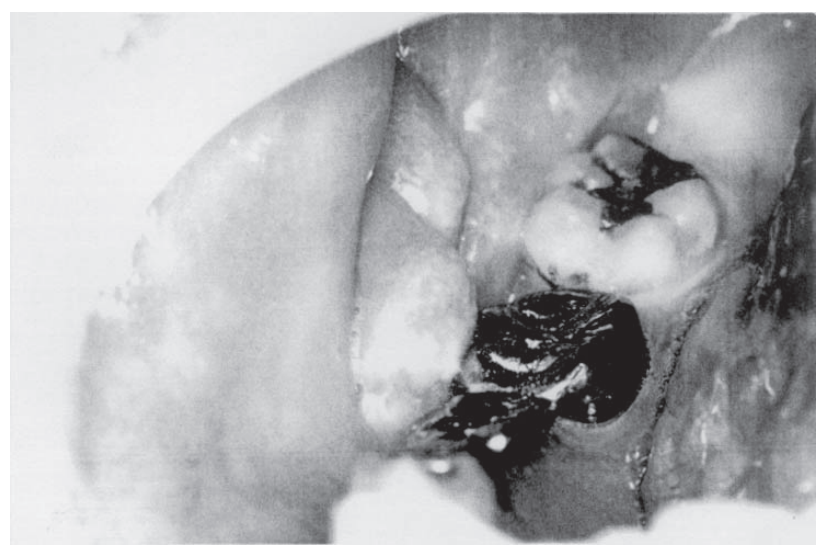

\section{Case report}

A 44-year-old Japanese male was referred to the Department of Dentistry and Oral Surgery at Jichi Medical School Hospital on February 16, 1998, by his family doctor. He complained of a nodular lesion in the right buccal mucosa. A medical history revealed hyperlipemia at age 40 years. The patient first noticed the nodular lesion about 3 months earlier, and the lesion gradually increased in size. Oral examination revealed two nodular lesions in the right posterior buccal mucosa (Fig. 1). Each of these lesions was soybean size, and they were covered by smooth epithelium, showing a diffuse pattern and some white in color. The lesions were elastic hard to palpation but were accompanied by no pain. There were no abnormal values in the general blood examination. Under a clinical diagnosis of benign tumor in the buccal mucosa, the lesions were surgically resected on February 23, 1998.

Fig. 1: Two nodular lesions in the posterior part of the buccal mucosa. 

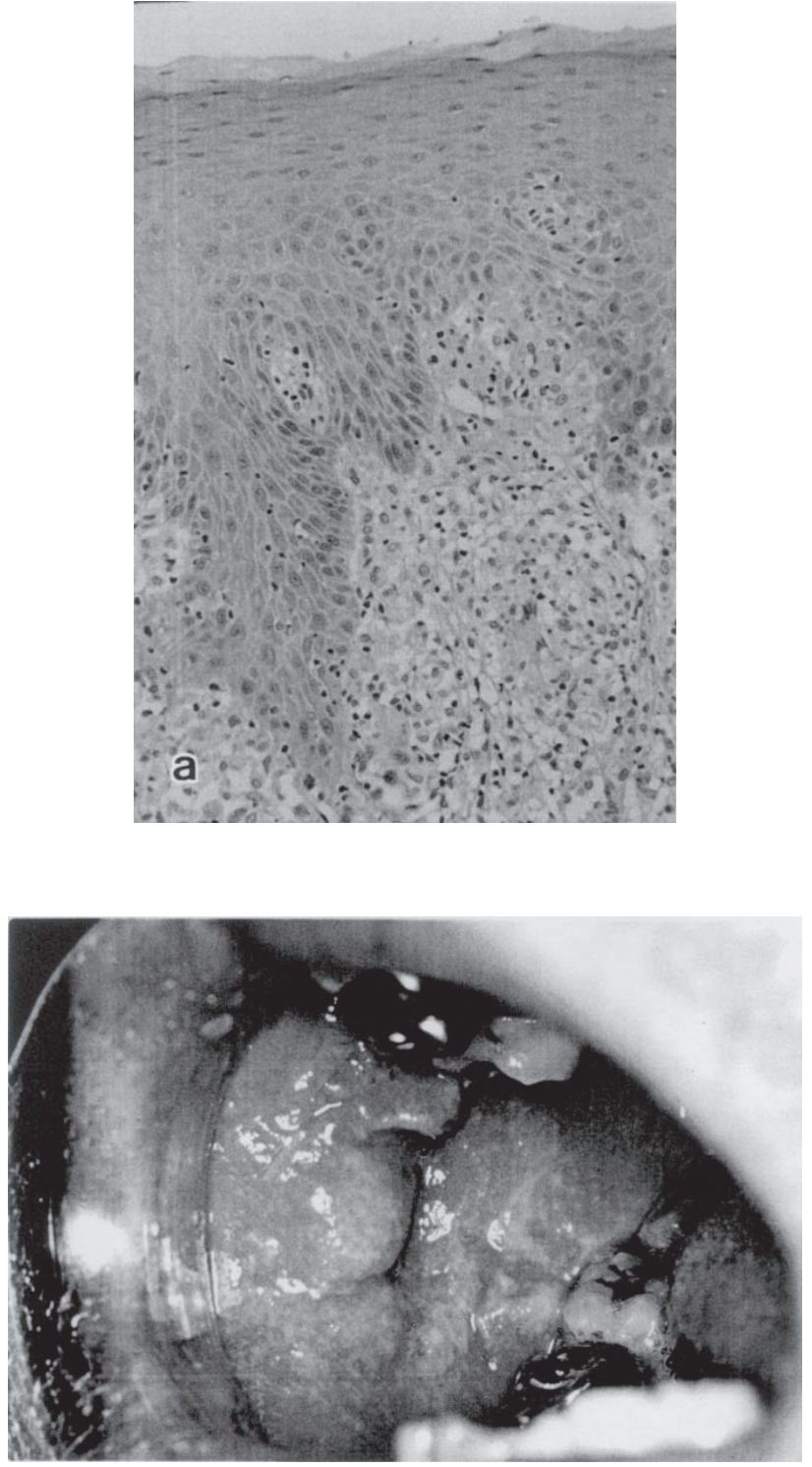

Histologically, inflammatory changes characterized by difuse lymphatic infiltration extended throughout the submucosal layer (Fig. 2a, b). Lymphedema and dilated lymphatics were also presented. Loose textured granuloma without caseating was multifocally observed at various depths in the submucosal layer. This granuloma consisted of relatively small nests and epithelioid cells composed of spindle or polygonal shaped cells with marked lymphatic infiltration. The caseous necrosis that was typical for tuberculosis was not observed and there was no evidence of mycobacteria. Covering squamous epithelium showed a mild degree of the hyperplastic change without atypia.

The pathological diagnosis was granulomatous stomatitis, although a differential diagnosis of Crohn's disease or sarcoidosis was recommended by the pathologist. Hematological examination, chest radiograph and endoscope were performed by the patient's family doctor. Although a small benign polyp was found in the colon, there were no abnormal findings in the digestive and respiratory organs.

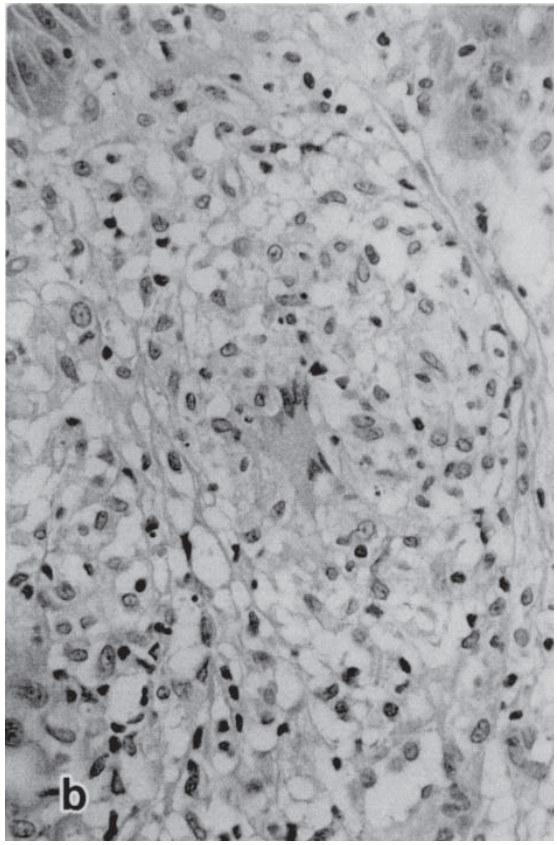

Fig. 2a: Microphotograph of the biopsy shows a non-caseating granuloma consisting of lymphocytes and polygonal shaped epithelioid cells in the subepithelial layer (HE, 50). Covering squamous epithelium shows mild hyperplasia.

Fig. 2b: This granuloma is very loosely composed of epithelioid cells (HE, 100).

Fig. 3: Swelling and redness of the buccal mucosa, and nodular lesions.

On January 7, 1999, about 11 months after his first visit, the patient re-visited our department, due to a recurrence of the nodular lesion in his right buccal mucosa. Oral examination revealed a diffuse swelling of the right buccal mucosa with two nodular lesions (Fig. 3). The mucosa showed redness accompanied by a mottled white appearance. The nodular lesions had an ill-defined margin and were located in the center of the buccal mucosa. These lesions felt elastic hard to palpation, and there was no spontaneous and oppression pain. Under suspicion of Crohn's disease or sarcoidosis, a detailed blood examination was performed, which included the erythrocyte sedimentation rate, serum albumin, alkaline phosphatase, calcium, magnesium, phosphate, serum iron, vitamin B12, folate and serum angiotensin converting enzyme. There was no evidence of malabsorption or serological evidence of Crohn's disease and sarcoidosis, and a chest radiograph showed no evidence of lymphadenopathy. Furthermore, endoscopy showed no lesions suggesting Crohn's disease. On January 13, 1999, the nodular lesions in the buccal mucosa were resected again. 

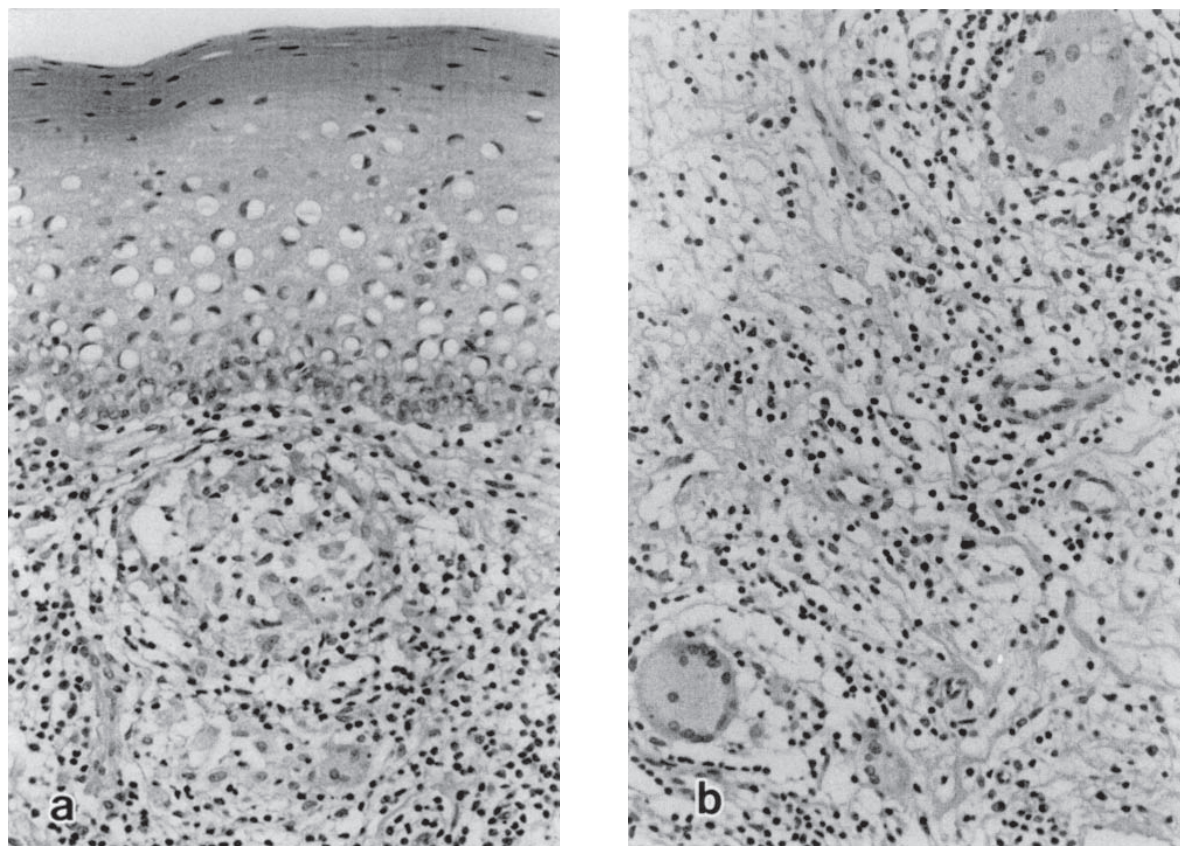

Fig. 4a: Microphotograph of the second biopsy is similar to histological findings of the first biopsy (HE, 50).

Fig. 4b: Within the granuloma there are two large, multinucleated giant cells. Lymphatic infiltration and lymphedema are also present in the submucosal layer (HE, 80).

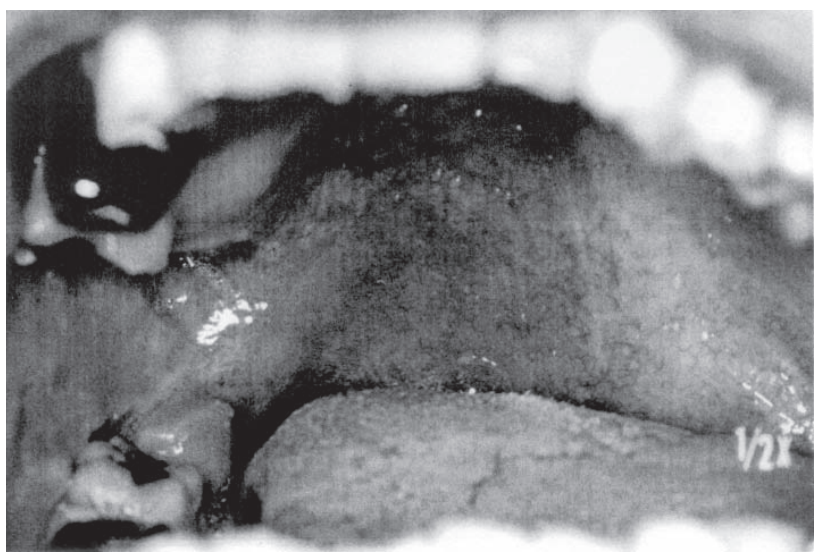

Fig. 5: Redness of the right palate.

Histopathological findings were the similar to that of the first biopsy (Fig. 4a, b). Within the granuloma there were large, multinucleated giant cells. Lymphatic infiltration and lymphedema were also observed in the submucosal layer.

We followed up the patient at intervals of 1 month. On June 8, 1999, redness of the right palate was observed, and because swelling and redness of the right buccal mucosa were also becoming more severe, topical corticosteroid was applyed to the buccal and palatal mucosa (Fig. 5 ). The redness of the palate gradually improved, and the swelling and redness of the buccal mucosa completely disappeared in 1 month (Fig. 6). On August 3, 1999, the lower lip suddenly swelled, but it too gradually improved in 1 month without any treatments (Fig. 7). Since these episodes, there have been no abnormal findings in the patient's oral cavity nor in his general condition, including his digestive and respiratory systems. We finally diagnosed the patient as having orofacial granulomatosis.

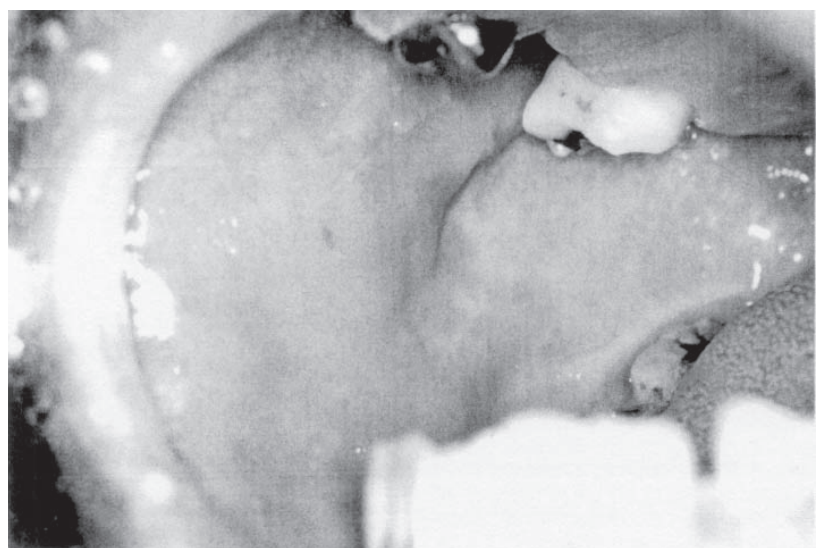

Fig. 6: The buccal mucosa 1 month after the application of topical corticosteroid.

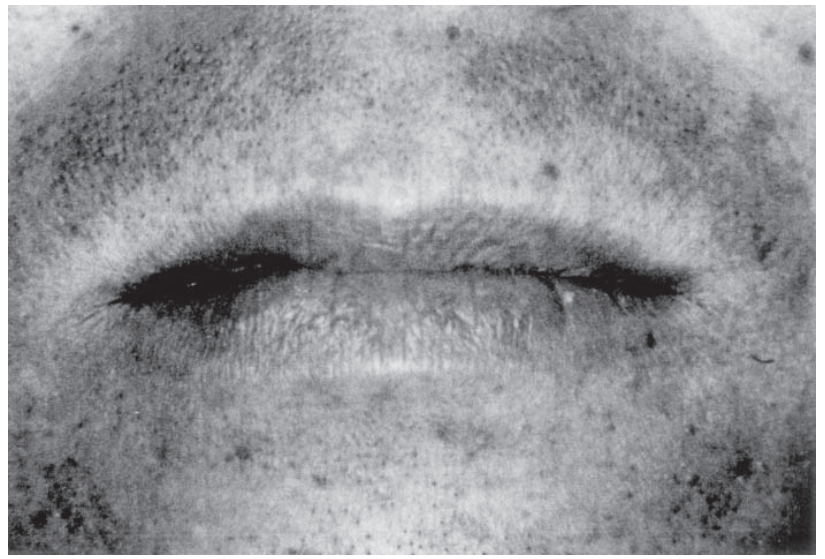

Fig. 7: Swelling of the lower lip. 


\section{Discussion}

The present patient was first clinically diagnosed as having a tumor in the buccal mucosa, but the resected specimen showed a non-caseating granuloma suggesting Crohn's disease or sarcoidosis. However, systemic and biochemical examination showed no evidence of Crohn's disease or sarcoidosis. Finally, based on the clinical features and pathological findings, we diagnosed the patient as having orofacial granulomatosis, even though the term orofacial granulomatosis is not commonly used in Japan.

The term orofacial granulomatosis, introduced by Wiesenfeld et al. (1), has been advanced to describe a clinical entity presenting with persistent swelling of the facial and/or oral tissues in association with histologic evidence of non-caseating granulomatosis inflammation. Orofacial granulomatosis includes Melkersson-Rosenthal syndrome and Miescher's chronic granulomatous cheilitis, as well as the localized orofacial presentation of Crohn's disease, sarcoidosis and mycobacterial infection (3-7).

According to Armstrong and Burrows (4), the age of onset is highly variable, with the median in most series in the second and third decades. The sex incidence is approximately equal. The predominant clinical features include labial and/or facial swelling in association with oral mucosal lesions. Labial swelling is most common, occuring in about $70 \%$ of individuals. Facial swelling occurs in about $50 \%$ and most commonly involves the lower half of the face. Oral mucosal involvement produces edema and erythema, particularly of the buccal and labial mucosa, and may give rise to an appearance of broad fold or cobblestoning. Oral ulceration is common (30-40\%) and, in addition to simple aphtae, may present as deep, presistent, and painful lesions. Facial palasy occurs in up to one third of cases in some series and is usually of a lower motor neurone type. Other features described included mucosal tags, cervical lymphadenopathy, lingual burning or swelling, disturbance in taste sensation, and gingival bleeding. Patients with Crohn's disease, sarcoidosis, or mycobacterial infection may present with similar orofacial manifestations $(8,12-17)$. Among them, the association of orofacial granulomatosis with Crohn's disease is well documented, and rates of 10 to $48 \%$ have been reported in various series (12-15). A few patients are reported to have specific intolerances to foods or additives such as cinnamaldehyde, carvone, peperritone, cocoa, carmosine, sun yellow, or monosodium glutamate, or to metal such as cobalt (9-11).

Histological findings consisit of edema in the laminapropria, perivascular mononucler inflammatory infiltrates, and non-caseating epithelioid granulomas with or without multinucleated giant cells $(2,6)$. In our case, pathology showed edema, non-caseating epithelioid granuloma and multinucleated giant cells. These granulomas are histologically similar to those found in Crohn's disease and sarcoidosis (8). To diagnose the patient with orofacial granulomatosis, hematologic and biochemical examinations to assess the possibility of malabsorption should be performed, and the serum angiotensin converting enzyme level should be estimated. Next, radiological examination should include a chest film, and endoscopy also should be performed to examine the intestinal symptoms or evidence of malabsorption. In the present case, there was no evidence of malabsorption, lymphadenopathy or intestinal lesion. Finally, the patch-testing for food and food additives remains to be clarfied. Careful diagnostic assessment is needed to distinguish orofacial granulomatosis from systemic diseases. Unfortunately, we do not have patch-testing procedures for food and food additives, so we could not test.

Armstrong and Burrows (4) reported that patients with minor symptoms may not require active treatment. Intralesional and systemic corticosteroids are generally effective, although often only partially so. Prolonged or repeated treatment may be necessary because of relapse on cessation of therapy with corticosteroids. Surgery may produce initial improvements in the apperance, but postoperative intralesional steroids are usually required and are often ineffective in preventing relapse.

The present case was treated with surgery and topical steroids, and there has been no relapse for 2 years after treatment. However, careful and long-term followup must be necessary for this patient.

\section{References}

1. Wiesenfeld D, Ferguson MM, Mitchell DN, et al. Oro-facial granulomatosis - a clinical and pathological analysis. $Q \mathrm{~J}$ Med 1985; 54: 101-13.

2. Editorial review. Orofacial granulomatosis. Lancet 1991; 338: 20-1.

3. Henry $\mathrm{CH}$. Orofacial granulomatosis: report of a case with decreased CD4/CD8 ratio. J Oral Maxillofac Surg 1994; 52: 317-22.

4. Armstrong DKB and Burrows D. Orofacial granulomatosis. Int J Dermatol 1995; 34: 830-3.

5. Rogers RS 3rd. Melkersson-Rosenthal syndrome and orofacial granulomatosis. Dermatol Clin 1996; 14: 371-9.

6. Cleary KR and Batsakis JG. Orofacial granulomatosis and Crohn's disease. Ann Otol Rhinol Laryngol 1996; 105: 166-7.

7. Clayden AM, Bleys CM, Jones SF, et al. Orofacial granulomatosis: a diagnostic problem for the unwary and a management dilemma. Case reports. Aust Dent $J$ 1997; 42: 22832 .

8. Rees TD. Orofacial granulomatosis and related conditions. J Peridontol 2000 1999; 21: 145-57.

9. Sweatman MC, Tasker R, Warner JO, et al. Oro-facial granulomatosis. Response to elemental diet and provocation by food additives. Clin Allergy 1986; 16: 331-8.

10. Pryce DW and King CM. Orofacial granulomatosis associated with delayed hypersensitivity to cobalt. Clin Exp Dermatol 1990; 15: 384-96.

11. Armstrong DK, Biagioni P, Lamey PJ, et al. Contact hyper- 
sensitivity in patients with orofacial granulomatosis. Am J Contact Dermat 1997; 8: 35-8.

12. Plauth M, Jenss H and Meyle J. Oral manifestations of Crohn's disease. An analysis of 79 cases. J Clin Gastroenterol 1991; 13: 29-37.

13. Field EA and Tyldesley WR. Oral Crohn's disease revisiteda 10-year-review. Br J Oral Maxillofac Surg 1989; 27: 11423.

14. Snyder MR and Cawson RA. Oral changes in Crohn's disease. J Oral Surg 1976; 34: 594-9.
15. Williams A, Wray D and Ferguson A. The clinical entity of oral Crohn's disease. Q J Med 1991; 79: 45l-8.

16. van Maarsseveen AC, van der Waal I, Stam J, et al. Oral involvement in sarcoidosis. Int J Oral Surg 1982; 11: 21-9.

17. Hildebrand J, Plezia RA and Rao SB. Sarcoidosis. Report of two cases with oral involvement. Oral Surg Oral Med Oral Pathol 1990; 69: 217-22.

(Accepted for publication November 22, 2001) 